\title{
Quasi-truth and incomplete information in historical sciences
}

\author{
(Cuasi-verdad e información incompleta en las ciencias históricas) \\ Jonas Rafael Becker Arenhart*, Vítor Medeiros Costa \\ Federal University of Santa Catarina
}

\begin{abstract}
Quasi-truth is a formal approach to a pragmatically-oriented view of truth. The basic plan motivating the framework consists in providing for a more realistic account of truth, accommodating situations where there is incomplete information, as typically happens in the practice of science. The historical sciences are a case in hand, where incomplete information is the rule. It would seem, then, that the quasi-truth approach would be the most appropriate one to deal with historical sciences, then. In this paper, we explore this possibility and use the historical sciences as a test case for the approach of quasitruth. Our claim is that, on what concerns historical sciences, the quasi-truth approach fails in two basic senses; first, by misrepresenting some cases concerning incomplete information, and second, by falling short of accounting for many features of incomplete information peculiar to historical sciences. We conclude that, despite its stated goals, quasi-truth must be either amended or substituted if the goal of a more faithful representation of scientific practice is to be achieved.
\end{abstract}

KEYWORDS: quasi-truth; truth; historical sciences; incompleteness; formal approaches to science.

RESUMEN: La cuasi-verdad es un enfoque formal para una visión de la verdad orientada pragmáticamente. El plan básico que motiva la posición consiste en proporcionar una descripción más realista de la verdad, acomodando situaciones donde se tiene información incompleta, como suele suceder en la práctica de la ciencia. Las ciencias históricas son un caso en la mano, donde la información incompleta es la regla. Parecería, entonces, que el enfoque de cuasi-verdad sería el más apropiado para tratar con las ciencias históricas. En este artículo, exploramos esta posibilidad y usamos las ciencias históricas como un caso de prueba para el enfoque de cuasi-verdad. Nuestra afirmación es que, en lo que concierne a las ciencias históricas, el enfoque de cuasiverdad falla en dos sentidos básicos; primero, al desfigurar algunos casos relacionados con información incompleta, y segundo, al no tener en cuenta muchas características de información incompleta propias de las ciencias históricas. Llegamos a la conclusión de que, a pesar de sus objetivos declarados, la cuasi-verdad debe ser enmendada o sustituida si se quiere lograr el objetivo de una representación más fiel de la práctica científica.

PALABRAS CLAVE: cuasi-verdad; verdad; ciencias históricas; incompletitud; enfoques formales de la ciencia.

* Correspondence to: Jonas R. Becker Arenhart. Department of Philosophy. Federal University of Santa Catarina. Campus Universitário Reitor João David Ferreira Lima. Trindade - Florianópolis (Santa Catarina-Brazil, 88040-900) - jonas.becker2@gmail.com - https://orcid. org/0000-0001-8570-7336

How to cite: Becker Arenhart, Jonas R.; Medeiros Costa, Vítor. (2021). «Quasi-truth and incomplete information in historical sciences»; Theoria. An International Journal for Theory, History and Foundations of Science, 36(1), 113-137. (https://doi.org/10.1387/theoria.21254).

Received: 2019, November 25; Final version: 2020, August 10.

ISSN 0495-4548 - elSSN 2171-679X / (C) 2021 UPV/EHU

This article is distributed under the terms of the

Creative Commons Atribution 4.0 Internacional License 


\section{Introduction}

It is not an overstatement to claim that philosophy of science is mostly concerned with physics. This has been changing in recent times, but the tradition is still very entrenched. Discussion on the nature of scientific theories have mostly taken into account the features of physical theories as a paradigm for the philosophical study of scientific theories. In this tradition, then, it is not a surprise that formal approaches to science have tended to focus on physical sciences, and, in general, in more mathematized sciences. This is illustrated by the classical debate about the most appropriate characterization of scientific theories, as for instance, in the dispute between the syntactic and semantic approaches (see Krause and Arenhart (2017, chap. 1), for an overview). There are few, if any, words on these accounts about non-mathematical theories, not to mention theories in softer sciences, such as historical sciences.

But even if we restrict ourselves to philosophical treatments of the physical sciences more specifically, the formal approaches to philosophy of science have recently undergone further important changes. More practice-oriented accounts of theories, models, and other important notions, have been advanced in the philosophical literature, and the relation between the philosophy of a particular science and its actual practice has been at the spotlight for some time now. Discussions on the appropriate understanding of models in science illustrate that (see da Costa and French (2003), for a whole book devoted to the subject). Certainly, this is an important progress on the philosophical understanding of science. However, given the lack of formal treatments for historical sciences, we lack, at least so far, even treatments about which one could complain that they do not take the practice properly into account. It is our belief that philosophical understanding may be obtained by such formal treatment of historical sciences too. In this sense, perhaps, in the lack of a developed formalism to deal with such sciences, one first step could be the appropriation of already existing formal approaches to other sciences, such as physics, as a guide for the formulation of specific formalisms for the historical sciences.

In this paper, that is precisely what we shall attempt to do. Instead of providing directly for a new formal treatment of historical sciences, built from the ground up, we shall attempt to both delimit what are some of the specific features of such sciences on what concerns incomplete information, and check how well a very widespread philosophical and formal approach to the sciences fares in dealing with them. That is, we put to test an already available formal account of science, and attempt to check whether it could deal with historical sciences if used as currently is. We choose precisely a formal approach that attempts to bring the scientific practice closer to the formalism, in the proposal of quasitruth, or pragmatic truth. The formal apparatus of pragmatic truth or quasi-truth (we shall use both as synonymous from now on), developed by Newton C. A. da Costa and collaborators, has as one of its main goals, to be an account of truth for science in a more faithful and realistic way, as it is currently practiced (see da Costa and French (2003), for the presentation of the general program). One of the most important aspects of scientific practice, according to the proponents of quasi-truth, lies in the fact that scientific knowledge is open or incomplete: in general we do not have all the relevant information about the phenomena we are investigating. As a consequence, there is incompleteness in the sense that scientific investigation is open to evolve in different directions, depending on the new evidence that is gathered as research progresses. 
Quasi-truth makes room for such incompleteness of scientific knowledge, being thus a more appropriate formalism (it is said) to represent science in philosophical investigations. The goal is to provide a concept of truth that is more flexible than the traditional one, which, it is thought, does not adequately capture important aspects of the scientific practice precisely because it excludes the mentioned incompleteness (again, see Krause and Arenhart (2017), for discussion on the traditional approaches to scientific theories). According to its proponents,

One of the main motivations for introducing this proposal comes from the need for supplying a formal framework in which the openness and incompleteness of information dealt with in scientific practice can be accommodated in a unified way. (Bueno and da Costa, 2007, p. 387)

A very similar claim about the aims of quasi-truth is to be found in Bueno (1999, p. 278). In another place, commenting on the idea that lack of knowledge means that science may evolve in distinct directions, due to distinct possibilities being open to scientific knowledge, da Costa and French comment that "[i]t is precisely this sense of partiality and openness that our approach attempts to capture and further explore" (da Costa and French, 2003, p. 14); that is, quasi-truth is an attempt at formalizing the idea that scientific knowledge is open and incomplete.

Now, it is not controversial that scientific theories traditionally dealt with by philosophers of science present some kind of incompleteness and partiality of information. Quasitruth has already been applied to the study of some of these theories, in a wide variety of dimensions (see da Costa and French (2003), for a discussion and general application of the pragmatic truth apparatus to the case of Bohr's model of the atom, and see Contessa (2007), for criticism of the approach in general lines). As one could guess, another area of knowledge that involves incompleteness and partiality of information concerns the historical sciences, whose features are still lacking appropriate investigation in the context of application of formal systems, either traditional, either non-classical, such as the apparatus of quasi-truth.

In order to provide the first steps to fill this gap, our goal in this paper is to study the prospects for applying the formalism of quasi-truth in attempting to accommodate incompleteness of information as it appears in the historical sciences. Discussing how quasi-truth fares in dealing with these sciences and some of their peculiarities on what concerns incomplete information, we shall be in a better position to evaluate the merits and limitations of the notion of quasi-truth in this field, and also, to motivate further developments that encompass the peculiarities of historical sciences that deserve to be taken into account in formal treatments that are still to be developed. Our claim is that quasi-truth faces difficulties in dealing with historical sciences, difficulties deriving from two main sources: on the one hand, there are difficulties directly related with the very formulation of the concept of quasi-truth, which will generate some obstacles in the application of the approach to any science, and on the other hand, difficulties that result from the peculiarities of the historical sciences, which the approach cannot clearly accommodate. Our emphasis shall be on the role incomplete information in historical sciences plays in causing difficulties to the quasi-truth approach.

The rest of this paper is structured as follows: in section 2, we present the formalism of quasi-truth as it is usually employed by da Costa and collaborators, so that the paper keeps 
self-contained. In section 3 we present, in general lines, what we mean by 'historical sciences' and how incompleteness and partiality may be found in these sciences. Having both the formal apparatus of quasi-truth and the features of historical sciences that need to be accommodated, we shall have the appropriate background information to evaluate whether the quasi-truth approach is able to appropriately accommodate the situations described in the historical sciences. This specific discussion that will take place in section 4 . We conclude in section 5.

\section{Quasi-truth: the basics}

In order to keep the paper self-contained, we present in this section the basic formalism of quasi-truth as presented in da Costa (1999), da Costa and French (2003), Bueno and da Costa (2007). Other formulations of quasi-truth are available, however (see Bueno and de Souza (1996), and Coniglio and Silvestrini (2014)). They are not equivalent to the one presented here, and they certainly require a separate discussion, which we shall not develop here. We restrict ourselves to the original approach by da Costa.

When we investigate a domain of knowledge, such as particle physics, the movement of planets, the history of a country, the history of an individual or of an artifact, we may use a structure to organize our knowledge of the entities involved and their properties and relations. In order to do that, we begin by stipulating a domain of entities $\mathrm{D}$, where we find the objects of our investigations, both concrete and ideal objects. In the case of historical sciences, we may be interested in particular individuals, documents, artifacts, the corresponding descriptions of facts taken as consensual, and other kinds of entities that mediate our knowledge of the past.

Apart from having picked a collection D of individuals, we also would like to study the relations holding between the objects in D. What happens frequently in real scientific practice is that there is a pervasive lack of information on what concerns property attribution to the target objects and also on the relations they bear among themselves. Many questions concerning such properties and relations remain open, and this openness must be somehow codified in a formalism attempting to reflect more closely the actual scientific practice. As it is known, in typical set theoretical analyses, a binary relation $\mathrm{R}$ over the elements of a set $\mathrm{D}$ (and we use it only to exemplify) is a set of ordered pairs $\langle x, y\rangle$ of elements of D that are related by $R$. As the typical definition goes, if $\langle x, y\rangle$ is in $R$, then $x$ is related to $y$ by $R$, and if $\langle\mathrm{x}, \mathrm{y}\rangle$ is not in $\mathrm{R}$, the relation does not hold for $\mathrm{x}$ and $\mathrm{y}$. There is no place for lack of information, or for incomplete knowledge; every relation is totally defined.

In the case of the quasi-truth apparatus, the notion of a relation (and, as a limit case, of a property) is made flexible to accommodate lack of information or of knowledge. This is codified by the concept of partial relation.

Def. 1 [Partial relation] An n-ary partial relation over a set $\mathrm{D}$ is an ordered triple $\mathrm{R}=\left\langle\mathrm{R}_{1}, \mathrm{R}_{2}, \mathrm{R}_{3}>\right.$, where $\mathrm{R}_{1}, \mathrm{R}_{2}$ and $\mathrm{R}_{3}$ are mutually exhaustive and exclusive over $D^{N}$ (that is, every element of $D^{N}$ is in exactly one of $R_{1}, R_{2}$ or $R_{3}$, and only one of them).

Formally, a partial relation is a set of three sets of n-tuples of elements of D; every n-tuple of $\mathrm{D}^{\mathrm{N}}$ is in at least one, and in only one of the sets. Intuitively, the definition attempts to 
capture the lack of information as follows: " $R_{1}$ is the set of $n$-tuples that belong to $R, R_{2}$ is the set of $n$-tuples that do not belong to $R$, and $R_{3}$ is the set of n-tuples for which it is not defined whether they belong or not to R" (Bueno, 1999, p. 279). That is, instead of having only a separation between those n-tuples that have $\mathrm{R}$, and those that do not have $\mathrm{R}$, we now also have a set of $n$-tuples for which information as to whether they bear $\mathrm{R}$ is lacking: the elements of $R_{3}$.

To put it in other words, a partial relation involves not only the positive and the negative extension of a n-ary predicate symbol, but it also has a third member whose purpose is to capture the epistemic limitations about the status of the information available for us on what concerns the entities in $\mathrm{D}$ (from now on, whenever we speak of the third member of the extension of a partial relation, it is to this kind of set we are referring to).

Def 2. [Partial Structure] A partial structure $\mathrm{E}$ is a pair $\mathrm{E}=\left\langle\mathrm{D}, \mathrm{R}_{\mathrm{i}}\right\rangle$, where $\mathrm{D}$ is the domain and $R_{i}$ is a family of partial relations over $D$.

That is, a partial structure generalizes the very notion of structure, and in the place of usual, total relations, it includes partial relations. This concept attempts to formally capture the incompleteness inherent in the practice of science, as claimed by Bueno and de Souza:

Da Costa's version of quasi-truth is put forward as a weaker notion of truth, appropriate for the 'partialness' and the 'openness' typically found in science and in everyday life. As a matter of fact, we hardly (if ever) have at our disposal complete information about any particular domain that we happen to be investigating. And, to a certain extent, this epistemic predicament can be formally represented with the introduction of a convenient concept of partial structure. (Bueno and de Souza, 1996, p. 188)

In our discussions from now on, we shall always suppose that for any partial structure $\mathrm{E}$, we also have an appropriate first-order language $\mathrm{L}$ of the same similarity type, so that $\mathrm{L}$ is interpreted in $\mathrm{E}$ in the usual way.

Now, recall that a partial structure $\mathrm{E}=\left\langle\mathrm{D}, \mathrm{R}_{\mathrm{i}}>\right.$ attempts to codify our information about the elements of the domain $\mathrm{D}$. As the intuitive interpretation of the partial relations mentioned before indicates, the third element of the extension of each partial relation codifies the open information, the one that is still requiring investigation to be settled. The partial relations may be extended in two distinct ways: once new information about the entities in $\mathrm{D}$ is found out, our ignorance decreases, so that each component $\mathrm{R}_{3}$ of a relation $\mathrm{R}$ may be displaced either to the component $R_{1}$, or else to the component $R_{2}$ (depending on the information obtained, of course). The problem is that some of these extensions may not be compatible with the information already available. We want to extend our knowledge by diminishing cases where knowledge is lacking, not to have new information that conflicts with already established facts. In order to shorten the number of extensions of $\mathrm{E}$ allowed in the appropriate way, we select a set $\mathrm{P}$ of sentences of $\mathrm{L}$ that are considered as true, in the classical, Tarskian sense of truth. These sentences, in the case of an empirical science, may involve descriptions of results of experiments, descriptions of directly observable facts, and (for those that are comfortable with that), scientific laws that one may be willing to countenance as true. The main purpose of $\mathrm{P}$, then, is to restrict the quantity of extensions of $\mathrm{E}$; an extension of $\mathrm{E}$ will be admissible only if it is a model (in the Tarskian sense) of $\mathrm{P}$ (Bueno 1999, p. 280; Bueno and da Costa, 2007, p. 388). 
Def 3. [Simple pragmatic structure] A simple pragmatic structure (sps) A is a triple $\mathrm{A}=\left\langle\mathrm{D}, \mathrm{R}_{\mathrm{i}}, \mathrm{P}\right\rangle$, where $\mathrm{D}$ is the domain, $\mathrm{R}_{\mathrm{i}}$ is a family of partial relations, and $\mathrm{P}$ is a set of sentences accepted as true in the Tarskian sense.

The result, then, is that what is more important for us are the simple pragmatic structures, not merely partial structures. This happens because an sps A involves a set of sentences restricting the extensions of A that are compatible with knowledge already available. Sentences of $\mathrm{L}$ shall be evaluated in a sps, not in a partial structure. In order to define how that is done, an intermediary definition is required:

Def. 4. [A-normal structure] Given a sps A for language L, a structure $\mathrm{B}=\left\langle\mathrm{D}^{\prime}, \mathrm{R}_{\mathrm{j}}, \mathrm{P}\right\rangle$ of the same similarity type as the structure $\mathrm{A}$ is said to be an A-normal structure if:

i) $\mathrm{D}=\mathrm{D}^{\prime}$ (the domains are the same)

ii) The interpretation of the individual constants of $L$ is the same in $A$ and in $B$ (they denote the same elements of the domain in both interpretations).

iii) Each relation in the family $R_{j}$ of $B$ is an extension to a total relation of a corresponding partial relation $R_{i}$ of $A$.

iv) $\mathrm{B}$ is a model of $\mathrm{P}$, in the Tarskian sense.

In other words, an A-normal structure is a total structure extending the partial relations of A to total relations which is also a model of P. Basically, a sps becomes a total structure when the third components of the extension of each partial relation becomes a member either of the first or of the second element of the extension of the same relation, always maintaining compatibility with P. Notice that every A-normal structure is a structure in the usual sense of a structure of classical first-order logic, with the proviso that it is also a model of $\mathrm{P}$ (then, the claim that the sentences of $\mathrm{P}$ are true in the Tarskian sense there make perfect sense). Before we move on, it is important to emphasize this: the first and second components of a partial relation on a sps remain fixed, only the third component of a partial relation is required to move when a total structure takes place by extending a sps; otherwise, $\mathrm{B}$ would not be an extension of $\mathrm{A}$, but merely another structure.

Having that in hand, we define quasi-truth for the sentences of $\mathrm{L}$. There are at least two definitions of quasi-truth available in the literature, which are clearly not equivalent:

Def. 5 [Quasi-truth version 1] A sentence $S$ of $\mathrm{L}$ is quasi-true in a sps $A$ in relation to an $A$-normal structure $B$ if $S$ is true, in the Tarskian sense, in $\mathrm{B}$ (see Bueno (1999, p. 280) and Bueno and da Costa (2007, p. 388), for instance).

Def. 6 [Quasi-truth version 2] A sentence $\mathrm{S}$ of $\mathrm{L}$ is quasi-true in a sps $A$ if there exists an A-normal structure B in which $S$ is true in the Tarskian sense (da Costa, 1999, p. 131; da Costa and French, 2003, p. 18).

Notice that definition 5 characterizes the concept of being quasi-true in a structure A relatively to an $A$-normal structure $B$, where $\mathrm{B}$ is held constant. The second definition of quasitruth, definition 6, on the other hand, does not fix a particular A-normal structure where $S$ will be evaluated, being it enough that at least one such structure exists. A consequence of this fact (in the second definition) is that a sentence $S$ describing some entities in the third component of the interpretation of a relation $\mathrm{R}$ may, let us say, be extended in such a way that these elements are shifted in some extensions to $\mathrm{R}_{1}$, and in other distinct exten- 
sions, to $R_{2}$. From this fact, it results that $S$ may be quasi-true in $A$, but we also have $\neg S$ being quasi-true in A. This, in certain ways, indicates incompleteness of information, according to the proponents of this approach (see the discussion of the particular case of Bohr's model of the atom in da Costa and French (2003, p. 105)). That is, given that information about an entity is incomplete, this reflects itself in the pragmatic truth approach by the fact that, under definition 6 , a sentence $S$ and its negation $\neg S$ may both be quasi-true in the same simple pragmatic structure A (see also Bueno (1999, p. 281)).

For a very simple example, consider a sps $A=\langle D, R, P\rangle$, where $D=\{0\}$, the set of accepted sentences $P$ is empty, and $R$ is unary, and has as its extension $R_{1}=R_{2}$ empty, and $\mathrm{R}_{3}=\{0\}$. That is, we have no information about the only member of the domain. Clearly, one total extension $B$ of $A$ consists in shifting 0 to $R_{1},\left(R_{1}=\{0\}\right.$, with $R_{2}=R_{3}$ empty) and another total extension, $B$ ', consists in shifting 0 to $R_{2}\left(R_{2}=\{0\}\right.$, with $R_{1}=R_{3}$ empty). In the first case, 0 has property $\mathrm{R}$, and in the second extension, 0 does not have property $\mathrm{R}$. Let us suppose, also, that the language $\mathrm{L}$ we are interpreting in $\mathrm{A}$ has 'a' as a singular constant denoting 0 , and that $\mathrm{R}$ acts also as a predicate symbol. Then, the sentence Ra is quasitrue in $\mathrm{B}$, but $\neg \mathrm{Ra}$ is quasi-true in $\mathrm{B}^{\prime}$. According to definition $6, \mathrm{Ra}$ is quasi-true in $\mathrm{A}$, and $\neg \mathrm{Ra}$ is also quasi-true in $\mathrm{A}$.

Definition 6, then, is responsible for establishing a close relation between incompleteness of information, as encapsulated in the informal explanation of the definition of a partial relation, and a kind of inconsistency (a sentence $S$ being such that $S$ is quasi-true and $\neg S$ being also quasi-true). Typically, in the quasi-truth formalism, a sentence dealing with incompleteness of knowledge or information, that is, one dealing with elements of the third component of a predicate, will be such that it is quasi-true, and its negation is also quasi-true in the sense of definition 6 . In this sense, it is said, the formalism of quasi-truth accommodates both the incompleteness and the inconsistency that is present in science (see da Costa and French (2003, chap. 5), and Bueno and da Costa (2007, p. 385, p. 390), for more on the inconsistency in science in the partial structures account). In this paper, our main focus will be the discussion of incompleteness, although some of our remarks may deal with the inconsistency too (for further discussion on the conflation of incompleteness of information with inconsistency in the quasi-truth approach, see Arenhart and Krause (2019)).

As a final remark, it is also important to stress that quasi-truth relates to truth in specific ways (we keep dealing with definition 6 here). First, quasi-truth is weaker than truth: if a sentence $S$ of a language $L$ is true in an $A$-normal structure $B$, then it is also trivially quasi-true in the sps A. But the other way around does not hold. This is easily understood: suppose that $\mathrm{S}$ is quasi-true in the sps $\mathrm{A}$, because there is an A-normal structure $\mathrm{B}$ in which it is true. But now, consider another A-normal structure $C$, where the target sentence $S$ is false. In this A-normal structure $\mathrm{C}$, the sentence $S$ is false, although it remains quasi-true in the sps A (see Bueno (1999, p. 281)). So, a sentence $S$ may be quasi-true, but still not be true. A similar reasoning illustrates that a sentence $S$ may be false, but not be quasi-false. Indeed, $S$ may be false in one A-normal structure B, but true in another A-normal structure $C$; this makes $S$ quasi-true, and not quasi-false. However, if $S$ is quasi-false, it is false in every total extension of $\mathrm{A}$, and thus, it is also false simpliciter. In this sense, falsity does not imply quasi-falsity, but quasi-falsity does imply falsity. 


\section{Historical sciences and incompleteness}

Our aim in this section is to motivate the claim that historical sciences exhibit cases of severe incompleteness of our knowledge, precisely the incompleteness that partial truth was aimed at to begin with. This is important for the purposes of this paper on two distinct fronts. First, because by bringing to light such features of historical sciences, we highlight the kind of incompleteness that formal accounts will have to deal with, if such formalisms are advanced to comply with important aspects of the practices of these sciences. Second, because it also brings the historical sciences to the fore as a field of application for those formal accounts of scientific knowledge already available in the literature that intend to encompass incompleteness, such as is the case of partial truth (as we have seen in the previous section); even though this particular account was not developed with the purpose of accommodating incompleteness of information or knowledge in historical sciences, a first attempt at dealing with incompleteness in historical sciences, as we shall discuss ahead, with use of this formalism, may enlighten the virtues and vices of this formalism, and indicate what kinds of features other formal approaches will have to encompass, if they are to succeed in this task.

Although a definition of any field of knowledge is a difficult, if not impossible, task, we shall indicate in general lines what we shall understand by historical sciences in the scope of this paper. Roughly, by 'Historical Sciences' we understand something similar to what Gallie (1984, pp. 472-493) defined as Genetic Sciences, although his definition starts from a specific conception of explanation in these sciences (cf. Gallie (1984, p. 480)); we, on the other hand, will characterize these sciences by considering how they describe their objects in time. Gallie deals with sciences that explain phenomena in historical time by giving necessary causes (which need not be sufficient) so that one can understand the conditions necessary for subsequent events to arise. This concept encompasses, besides historiography, parts of biology, geography, geology, etc. The difference from our definition is that ours focuses on the description of objects in historical time; which has the advantage of working for these (so-called "historical") sciences even if one or more of them do not explain ("tell the sufficient causes of') phenomena, but only describe them. Indeed, under the nomenclature of 'Historical Sciences' we have named a set of sciences or scientific sub-areas (such as Historiography and Natural History) that explain and describe objects past or present in historical time (a type of classical, non-relativistic time). They physically contain irreversible processes such as the process of aging, acculturation in a society and the process of species extinction, given in the time arrow of thermodynamics, which states that phenomena always occur with increasing entropy (Lopes (1992); Burgess (1984, pp. 130-131)).

A practical way of introducing the description-explanation distinction into historical sciences is by observing, for example, that a historian describes a past phenomenon when he answers a what-question, "what happened at such a time?", and he explains a past phenomenon when answering a why-question, "why did it happen like that?" or "why did it have to happen like that?" (see Little (2010, pp. 5-6)). We can give some examples of irreversible phenomena located in historical time (at different levels of precision) that are the object of the descriptive study of historical sciences:

- the extinction of dinosaurs around 65.5 million years;

- the assassination of Julius Caesar on March 15th, 44 BC. 
From a descriptive point of view, these two examples are statements that answer "what happened to the dinosaurs around 65,5 million years ago?" and "what happened to Julius Caesar on March 15th, 44 BC?” Another question would be to ask why Julius Caesar was murdered or why the dinosaurs were extinct. But these last two kinds of questions will not be the focus of our analysis. From an epistemological point of view, for this article we will highlight only one influential conception and particularly important for our debate: the actualism (Currie, 2019, pp. 6-7), which consists in the conception that knowledge of the past depends on the present and any secure statement about the past needs to refer to some present source. In this sense, with the addition of others sources about the past or the implementation of new methods to examine them, historical scientists are authorized to review their statements about the past. Thus, limiting ourselves to consider the descriptive aspect, we can assume that a historical theory about what happened at a time $t_{n}$ is a set of candidate propositions to be true at $t_{n}$ based on the current evidence from sources at the actual time $t_{0}$. We will later adapt this definition for pragmatic structures.

The definition of historical science that results from these considerations, then, may be stated as follows:

Def. 7 [Historical science] A science is historical when (I) its propositions (like "it was the case that p") are instantiated in historical time (non-relativistic time) and (II) it epistemologically access the past through either evidence and testimonies in the present or extrapolations from comparisons of other objects of the present with the assumption that the forces of nature that govern past phenomena are identical to the forces of nature at the present time (actualism).

Remark. In addition, the heuristic principle of gradualism (Currie, 2019, p. 6) can be added according to which social or natural phenomena (such as the appearance of a mountain, a speciation, a formation of a new natural language or the spread of a religion) generally do not occur abruptly, but incrementally.

The above definition seeks to satisfy, for different disciplines (with different methodologies), what Arthur Danto (1985[2007], pp. 24-25) called the minimum condition for a descriptive historical knowledge: "the very least that historians do is to try to make true statements, or to give true descriptions, of events in their past", i.e., the knowable past in relation to their present (Danto 1985[2007], p. 37).

In this sense, a logical formalism that intends to formalize the state of art of the historical sciences needs to capture the development of these sciences, covering their lack of information (epistemic incompleteness) in historical time. This is made explicit by considering the next definition:

Def. 8 [Epistemic incompleteness] We say that an object is epistemologically incomplete when knowledge of any of its properties is lacking.

Remark. Factual ignorance understood as the absence of knowledge could be divided into three subcategories (Martínez-Ordaz, 2020): absence of factual knowledge (lacking knowledge of either facts or the truth of specific propositions), absence of objectual knowledge (not knowing a particular object) and absence of procedural knowledge (not knowing how to do certain tasks). The criticisms that we will present in this article raise problems that affect the three subcategories, although the examples that we will use are more directly linked to the first and the last case of this classification. In historical sciences, the first case applies 
to moments when we do not have enough information to affirm something about an object, sometimes even because it is impossible to reconstruct a part of the past, as sometimes, for example, when trying to discover whether a dinosaur is male or female. The second case applies when we don't know whether an object-like Homer, Socrates or Shakespeare-really existed according to the reports. The third case, when, for example, we have before us an artifact-like a Greek statue-or a text with useful information about the past, but we do not know how to extract it. And we would add: there are cases in which the documentation may be false (in this respect we will mention the famous case of the Donation of Constantine). All the inadequacies that we will discuss shortly do not seem to be exclusive to the historical sciences, that is, exclusive to factual propositions indexed in historical time about which we are ignorant. But some are perhaps more serious in the historical sciences because it generally takes a long time to reach broad consensus on a fact, which reflects the particular epistemic incompleteness of the past as, in principle, there are very few direct sources on certain events.

For example: even when we find a complete fossil record of a dinosaur, we still cannot determine certain facts about it, such as whether it is male or female, except on rare occasions such as when the creature was pregnant at the time of fossilization (see Wilson, 2008). This does not mean that this information did not exist, in fact, the dinosaur was either male or female, it is, after all, an ontologically complete object, but the information has been lost in the current world (present moment), making verification inaccessible of that dinosaur property.

In this sense, the historical object is epistemologically incomplete, but it is ontologically complete, different from fictional objects that have ontological (not only epistemological) incompleteness. We will never be able to know, for example, how many children Lady Macbeth had in the fictional world created by Shakespeare, but this is because the relevant facts about which we could get this information never existed, and not because of current epistemic limitations or the loss of that information from the present moment (Doležel, 1998, p. 795).

In this way, a complete object and an incomplete object within historical time appear from two basic premises: (i) the real past is ontologically complete; in this sense, every single proposition about the historical object is already true or false; but (ii) the worlds of historical objects are at least epistemologically incomplete. This distinction between epistemic incompleteness in opposition to ontological incompleteness is precisely what is at stake also in the quasi-truth approach, which is also said to deal only with epistemic incompleteness (see the remarks in Bueno (1999, p. 279), explicitly about this distinction). And that's also why Doležel (1998, p. 795) understands that the incompleteness of historical worlds is epistemological; the incompleteness is a result of the limitations of human cognition. Doležel presents, to illustrate his point of view, a metaphor of Paul Veyne: "History is a palace whose full extent we do not discover... and of which we do not see all the suites at once" (as quoted in Doležel (1998, p. 795)).

However, the reason we say "at least" in (ii) is because historical pasts can be viewed constructively/positively or negatively. From a constructive/positive point of view, scientists as historians and naturalists construct certain cognitive models (here called historical past/worlds) to capture part of the real past; however, it seems impossible that a model will ever be achieved that fully (in the smallest details) captures the real past (as it was) in its ontological completeness, so it can be concluded that models of the historical pasts are onto- 
logically incomplete. In other words, although the "real past" was ontologically complete, it can be seen as no longer complete, at least on occasions when it is not about epistemic limitations, but in fact the impossibility of knowing something about it; this seems to be the case with the sex of certain dinosaurs. On the other hand, from a negative point of view, we imagine that there must be as much historical pasts as possible to the information we have about the past: suppose that at present instant $t$ we have no information about the past concerning an event or object, in which case we will have a huge (but finite) number of historical past/worlds accessible to the present; and in this as in any case with more than one historical past we will consider that each historical past is "potentially ontologically complete", in the sense that any of the historical pasts is the real past, although we do not know which one.

In the two explanations of (ii) it is possible to understand a certain dynamic progress in the historical sciences. In the first case, progress is made by building ever richer historical pasts by considering what we know about the real, and thus approaching the real as much as possible. In the second case, progress is made by eliminating those historical pasts that at some point contradict the data we have today $(t)$ about the real past. We can conclude that in both cases the epistemic failure of scientists is due to the lack of information about the real past; either to eliminate possible past models or to build a model analogous to the real past.

The quasi-truth approach seems prima facie to be an appropriate tool to capture the lack of information in the historical sciences, as we have been describing them. This appears to be quite straightforward, and may be understood roughly as follows. Let us fix an instant $t$ as the present instant, and let us suppose that we have a set of past propositionspropositions of the kind "it was the case that" $P a, P b, \ldots, P \pi$, describing an event or object in the historical past. It is also assumed that scientists in $t$ have epistemic incomplete access to their past worlds, so that there are sentences $S$ interpreted in our sps of $t$ about which there is not enough information to gauge whether they are true or false in their past (epistemic incompleteness), although in fact they have to be either true or false there (they are ontologically complete). These sentences, concerning objects or events in the past, will have to benefit from partial relations in order to codify the incompleteness of our actual information about such objects; in fact, the third component of the extension of a relation will have to accommodate the entities of the past about which our information is lacking. In this sense, the quasi-truth approach could be seen as suited not only for dealing with incompleteness in the empirical sciences, as typical applications of this formalism have it, but also as able to capture the epistemic indeterminacy of certain sentences about the past. Notice also that at least in principle, it is not mistaken to think that the fact that a sps may be extended into distinct total structures reflects the distinct ways our information about the past may be completed. These total extensions are the distinct worlds of the past object or event, and certainly, due to the assumption of ontological completeness of historical objects, only one of them is the real past. The trouble, of course, is that we don't know which is the real one.

Now, even though the approach seems to work perfectly well for lack of information or incomplete knowledge in the historical sciences, some questions remain as to the adequacy of the formalism to capture the important sense in which historical sciences are said to be incomplete. We shall discuss these in the next section. By bringing to light some of the difficulties in accounting for incompleteness in historical sciences by the pragmatic 
truth formalism, we hope to indicate how new formalisms may be developed which account for these features of historical sciences too. That is, bringing those difficulties to light is a first step to the development of new formal tools.

\section{Combining quasi-truth and historical sciences}

From what we have already presented, both from the conceptual machinery of quasi-truth and from the nature of historical sciences, the first impression may be that the concept of quasi-truth, with its intended aim of accommodating incompleteness in knowledge in a quite general setting, adequately captures also the openness and incompleteness that is specific of historical knowledge and information. It is enough, it may be thought, that we use partial relations to codify what remains open on what concerns our knowledge about the past, employing the third component of the extension of a partial relation for that purpose. However, the quasi-truth formalism has some peculiarities that generate diverse difficulties when one wishes to deal with important aspects of incompleteness involved in historical sciences. These difficulties, it seems to us, make the formalism of quasi-truth as somehow only a first step in dealing with these sciences, not really appropriately representing the intended incompleteness in all its specifics. All of that puts some limits to the expectations that the quasi-truth approach could be really the best choice on a formalism to deal with incompleteness and partiality of scientific knowledge. We shall discuss some of these difficulties now.

To simplify the treatment and discussion of some of the difficulties of the notion of quasi-truth in its connection with the historical sciences, we have separated the problems in two large groups, which are not completely exclusive (they are treated in the next two subsections). The separation reflects the kind of difficulties met by the formalism in accounting for specifics of the historical sciences:

a) inadequacies of the formalism, specific and general;

b) limitations of the formalism.

As the names indicate, the formalism may be inadequate to capture some characteristics of historical sciences, somehow misrepresenting important features of these sciences, but it may also be limited, leaving aside important features of these sciences. In the second case, concerning limitations, we indeed have an opportunity to try to amend the formalism to accommodate them, although it is not always clear how to do that in a satisfactory manner, and it is not clear that it could be helpful in every situation, given that the inadequacies will remain. Let us see.

\subsection{INADEQUACIES OF THE FORMALISM}

We next divide each particular aspect concerning the inadequacy of the formalism of quasitruth in distinct topics. The topics are advanced, and arguments, explanations and illustrations are presented, in order to make our case clearer.

i) Every proposition about which we have no knowledge is quasi-true, and its negation is also quasi-true. This makes room for a quite unrestricted assumption of 
plausibility of every proposition about which we have no knowledge, irrespective of whether it has any kind of plausibility against the background knowledge already assumed. Let us check.

The formalism of quasi-truth, through the definitions of quasi-truth (both definitions 5 and 6, so that it is irrelevant for this point which definition one adopts), makes equally quasi-true every sentence about whose status we have currently no information. In other words, if we do not have information about the truth of an atomic sentence $\mathrm{Pa}$ (where ' $\mathrm{P}$ ' is a unary predicate symbol and ' $a$ ' is an individual constant), and if we do not also have information about the truth of $\neg \mathrm{Pa}$, both result quasi-true, because there is a normal structure in which one of them is true in the Tarskian sense, and there is another normal structure where the other is true in the Tarskian sense also. This is a simple consequence of the fact that, for every formula dealing with the member of the third component of a partial relation, we can extend the original sps in two distinct directions, according to the definition of quasi-truth (see our discussion in section 2).

This scenario, although it is a simple fact concerning the current formalism of quasitruth, however, does not reflect the situation of lack of information or knowledge in historical sciences (and also neither in the physical sciences, we should say; see also Arenhart and Krause (2019)). In general, even when some extensions of a sps are legitimate from a formal point of view, they are not taken into account as plausible or interesting by historians, and they would neither receive the title of true or quasi-true. The formalism lack the means for separating extensions that are more plausible or more interesting from those extensions that are less plausible or less interesting. The only constraint on the allowed extensions is consistency with accepted data, and this constraint is way too liberal; mere consistency with the accepted data (the sentences in the set $\mathrm{P}$ ) is not enough to discriminate between these distinct situations. Some historical possibilities are more interesting and have much more weight than others in the course of investigation. The formalism, however, is blind for that, and this is something that a more faithful formalism should take into account, if it is to portrait faithfully the situation. That is, for a formalism to allow for a better representation of the situation in practice, one would need to be able to rule some consistent, although implausible, extensions.

As an example of how such feature of quasi-truth is in conflict with actual practice, let us consider a case in historical science again. We mention one that has inspired the literature, such as the famous poems by Fernando Pessoa and Oscar Wilde: the death of Antinous-the favorite and beloved catamite of the roman emperor Hadrian-in October of 130 d.C., and which is a case debated up to our days in historiography (so that our knowledge about it is clearly open yet). It is known that Antinous died from drowning in the Nile, close to the place where, afterwards, Hadrian gave name to a city in his homage: Antinopolis. Among the most plausible interpretations for the death of Antinous, we find one claiming that Antinous, due to revolts in the empire and health problems of Hadrian, sacrificed himself in a religious ritual, of which both he and Hadrian were members, and which had, as one of its consequences, perhaps, the later deification of Antinous and his identification with Osiris in sculptures. In the literature, the favorite interpretation of these events, however, is different. As Fernando Pessoa and Oscar Wilde illustrate, it is suggested that Antinous could have committed suicide for romantic reasons. A further attempt to explain the death, much less plausible, though, is that Antinous could have been murdered 
for political reasons, although his status and origins did not represent any kind of political threat to the emperor. According to the formalism of quasi-truth, the problem we are pointing to in this section is that these interpretations would all result equally quasi-true, and they would have the same weight among themselves. Also, from an epistemic point of view, historians would have to equally believe in the three interpretations (each of which would be characterized as pragmatically true), which would make impossible the distinction which really happens in the scientific community, according to which some interpretations are more (or less) plausible than others. Note that the problem remains in cases where it is much more likely that a historical entity like Socrates has existed than it does not, although his non-existence is not a completely implausible hypothesis, only improbable.

This complete freedom for every sentence describing open knowledge to be quasi-true, along with their negations, seems to indicate that the representational apparatus is somehow conflating open knowledge with contradiction. As our example in section 2 illustrated, this clearly happens for sentences dealing with situations about which we are ignorant. However, as we have been discussing, what we typically do in such situations is not to embrace every possibility, but to rank them according to their plausibility. In cases where we do not have any reason to believe one more than the other, we seem to suspend our judgment, not to accept that both a sentence and its negation are quasi-true. As Lo Guercio and Szmuc have put it, in another context:

\begin{abstract}
"in cases in which there is equally strong conflicting evidence, we claim, one must suspend judgment. What is the epistemic benefit of that? Of course, suspending judgment is not something that will contribute to the goal of accepting what is true. However, by suspending judgment one avoids accepting what is false. In the cases at hand, in which the evidence makes $A$ and $\neg A$ equally likely, following the evidence is not helpful in order to achieve the goal of accepting true propositions, so the best we can do is to avoid accepting false ones. By suspending judgment we do precisely that." (Lo Guercio and Szmuc, 2018, p. 164)
\end{abstract}

Besides having no discrimination power between more or less plausible extensions, a related problem that should be mentioned for the quasi-truth approach is as follows: sentences concerning the subject under investigation but on which we have no theoretic interest and about which we have no current information, are always settled in total structures, even if we have not obtained any information about them. That is, their alethic status is improved for free, as it were, just because a structure needs to be total. This is related to the fact that an extension needs to be a total structure, so that every time one extends a structure to accommodate some kind of evidence, all other sentences that were open get settled too, as a kind of bonus. This is also related with the next point.

ii) Changes from ignorance states to knowledge states are always carried over in a single step: from a sps where knowledge is incomplete to a total structure where it is totally determined (this issue relates to what has been discussed in (i)).

We could put this point as follows: there is no progressive growth of knowledge. The formalism does not adequately capture the situation described by da Costa and French presented before (2003, pp. 13-14), because it does not take into account that the discoveries of new evidence proceeds on a step by step basis. The progress of knowledge on the context of quasi-truth, however, is always carried over in a single step, through a unique extension. 
One possible way to correct this problem would be to change the formalism and allow the existence of simple pragmatic structures involving also merely partial extensions of the partial relations, so that a partial structure could be only partially extended, being transformed from a partial structure into another partial structure (keeping P intact), through the determination of only part of the lack of information. If that could be done, however, we would still have the difficulties with the revision of knowledge (which is still not accounted for, as we have already discussed), and the definition of partial truth would still have to take into account only total structures extending the actual sps.

A good illustration of this point may also be seen from our previous example of the Greco-Roman statues. In this case, what really happened was that only gradually we came to know that the Greco-Roman statues were, most of them, highly polychrome. At a first stage, some exemplars were found with partially preserved coloration, and fragments of texts were analyzed where technical and/or aesthetic aspects of the painting of statues were mentioned, and, as a final stage, the use of a technique called raking light, which uses ultraviolet light, contributed to determine the scale in which polychromatism predominated in Greco-Roman painting of sculptures, and contributed as well in the reconstruction of the colors they effectively had. The intermediary steps which gradually led to our actual stage, where a consensus is reached in historiography, may be understood as moments in which an interpretation was gradually becoming more plausible than others, until it finally achieved the status of a consensus (or something we could call "historical knowledge").

iii) The formalism identifies lack of information about a sentence $S$ with the possibility of S. We cannot have lack of knowledge or lack of information of necessary sentences; everything we ignore is possible.

In the formalism of quasi-truth, the ignorance about the alethic status of a sentence $S$ (i.e. whether it is true or false) may be enlightened in an alternative way, through the use of alethic modal concepts and a correspondence between quasi-truth and modalities. Basically, the idea is as follows: a sentence about which we currently do not have information (i.e. we are ignorant of its truth value) may be understood as somehow eligible for being true when further information is obtained, which makes it a possibly true sentence. Something similar happens to $\neg S$, that is, given that we do not have information about the truth value of $S$, we also do not have information about the truth value of $\neg S$, so that if $S$ could possibly be true when new information comes, the same may be said of $\neg S$ (see also our previous remarks relating openness and inconsistency).

This relation between quasi-truth and alethic modalities gets clearer with the modal interpretation of quasi-truth, as suggested by da Costa ((1999, pp. 135-136), for a fuller development see da Costa, Krause and Bueno (2006, pp. 851-858)). If we consider a fixed sps $\mathrm{A}$ as playing the role of the actual world, and if we treat its total extensions (the corresponding A-normal structures) as the possible worlds accessible from A, a modal interpretation of quasi-truth (through the possible worlds semantics) is immediate. An A-normal structure counts as a world accessible from $A$, and a sentence $S$ is quasi-true in A if and only if it is possible in the modal translation (in the corresponding sense that it is true in the Tarskian sense in a total extension of A). The system advanced to regulate the behavior of the modal operators is $\mathrm{S} 5$.

In this context, if this kind of correlation between total structures and possible worlds is made, and a corresponding correlation between quasi-truth and possibility is made, and 
given that sentences $S$ representing lack of information or of knowledge are those sentences for which there is an extension in which $S$ is the case, and another extension in which $\neg S$ is the case, then, in the modal idiom, our knowledge is open as to the status of $S$ when both $S$ is possible and also $\neg S$ is possible, as we commented in the beginning of this topic. What results from this association of quasi-truth with possibilities is that there is an equivalence between necessary sentences and those for which it is already known that they do hold or do not hold in the sps A under examination. In other words: whenever we ignore the information relevant for deciding the status of a sentence $S$, it is implied that both $S$ and $\neg S$ are possible. Also, if a sentence $S$ is necessary, it must be true in every extension of the sps A in which we are evaluating $S$, and in particular it will be true in the actual world.

This is particularly problematic in the case of identity statements. Ever since Kripke's work on rigid designation, it is common to allow that some propositions are necessary, although our knowledge of them may be a posteriori. A particular example would be the famous identity 'Hesperus $=$ Phosphorus'. That means that although the statement is necessarily true, there may be a time in which we don't have the information of which is the case. However, given the identification between possibility and quasi-truth, in that time in which knowledge is open, both 'Hesperus $=$ Phosphorus' and ' $\neg$ (Hesperus $=$ Phosphorus)' are possible. However, given the necessity of identity, one of those statements is simply impossible. In this scenario, we fail to capture the lack of information. That is, the correlation between modalities and lack of information seems to conflict with other facts known about modalities.

Another curious fact of this modal analogy concerns the consequence that everything we think we know results necessary. This is evidenced when one notices that a sentence dealing with the first or the second component of the interpretation of a predicate or relation symbol in a sps A cannot be changed when one shifts to the extensions of A (again, see our comments in section 2). Everything that is taken for granted as known (i.e. that an n-tuple of elements of the domain is in the extension of an n-ary predicate, or that it is not in such an extension) becomes a necessity. All accepted sentences become necessities (that includes the sentences in P). This clearly goes against the fact that most of our actual information, even those that eventually are accepted as true, are contingent (the examples above of dramatic changes in historical sciences also illustrate this). So, if P contains a sentence such as 'Socrates was Plato's teacher', this sentence results necessary, which is highly counter-intuitive (to say the least).

Notice that another consequence of the formalism is that contingencies, in the usual sense of the term, are impossible. According to the usual definition of contingency, $S$ is contingent iff $S$ is true, but it is possible that $\neg S$. When it is the case that $S$ in the actual sps where sentences are evaluated (the one selected as the actual world in the modal correspondence), any total structure in which $\neg S$ is the case is blocked (that is, $S$ becomes necessary, as we mentioned).

Perhaps one way to avoid the problem would be to shift from an alethic reading of the modal operators to an epistemic reading. A quasi-true sentence that is not absolutely true, that is, a possible sentence, is just believed to be true, and then, it seems, some of the worries with contingencies and necessities disappear. Sentences that are open to change are merely believed, those that are known for sure, however, are those whose statuses won't change anymore.

That solution, however, comes with its own costs. The first one concerns the epistemic status attributed to the sentences in $\mathrm{P}$, and also those sentences involving the first and second components of the extension of predicate and relation symbols. Given that they must 
remain fixed in every total structure, they count as known for sure and forever. That is, as we have already commented in section 2 , some of the sentences in a language interpreted in a pragmatic structure $A$ will have unchangeable truth values, and will count as known with certainty in this interpretation. This certainly goes against the fallibilistic character of most of science. Our examples, mentioned before, provide evidence that even the most confidently entrenched beliefs may be revised some time.

One could attempt to avoid these difficulties by shifting to a modal system weaker than S5. Some of the accessibility relations would no longer be available. However, notice that this would be of very little comfort. All that is needed for the above objections is that from a structure $\mathrm{A}$ one may access all the total extensions of $\mathrm{A}$, which is needed for the definition of quasi-truth. One does not really use all the properties of accessibility of S5 (like symmetry or reflexivity), so nothing special depends on that. However, changing to a weaker system may generate further problems: the so-called logic of pragmatic truth, the logic governing the behavior of quasi-truth, is precisely Jaskowski's logic, which is translatable to S5 (again, see da Costa, Krause and Bueno, 2006, pp. 851-858). Given that a sentence $S$ is quasi-true in a sps $\mathrm{A}$ if it is possible in the corresponding modal interpretation, a change of logic would simply require a change in the concepts of quasi-truth.

A second problem concerns the sentences that deal with the third component in the interpretations of predicates and relations. They attempt to reflect the openness of knowledge, and as a result, we may merely believe in them, but not know them, in the sense of epistemic logic. That seems a pretty sensible thing to do, that is, when there is lack of information, we are left with mere belief, not with full knowledge. However, as a result of the definition of quasi-truth, for any such sentence $S$, we end up believing in $S$ and also in $\neg S$, for the mere fact that we do not have information about $S$. But that seems to get things wrong. Once we don't have information about the alethic status of a sentence $S$, we don't typically believe that $S$ and also that $\neg S$ are the case; we are more well-advised to not believe in any of them, until further information appears. That is, instead of $(\mathrm{B}(\mathrm{S}) \& \mathrm{~B}(\neg S)$ ) (belief in $S$ and belief in $\neg S$, which would constitute a violation of an internal version of the Law of Non-Contradiction (LNC)), ${ }^{1}$ we are better off holding that $\neg \mathrm{B}(\mathrm{S})$ and $\neg \mathrm{B}(\neg \mathrm{S})$ (a denial of a version of the Law of Excluded Middle (LEM)). That is, lack of information, if it is to be understood in epistemic terms, should not be conceptualized in terms of a violation of the internal version of LNC, but rather in terms of a violation of the internal version of LEM. This is clear from discussions between paraconsistency and paracompleteness, such as in Carnielli and Rodrigues (2019). According to this approach, $(\mathrm{B}(\mathrm{S})$ $\& \mathrm{~B}(\neg S))$ represents excess of information and not lack of information. The quote by Lo Guercio and Szmuc (2018) presented before illustrates precisely this situation. For further insistence in this point, we follow them when they affirm:

"In contrast, it is not clear what would be the epistemic gain of accepting a contradiction. Accepting a contradiction not only does not contribute to the goal of accepting true things but it conspires against the goal of not accepting false ones, since by accepting a pair of contradictory propositions one is guaranteed to accept something false." (Lo Guercio and Szmuc, 2018, p. 164)

1 The so-called 'internal' version of $\mathrm{LNC}$ reads $\neg(\mathrm{B}(\mathrm{S}) \& \mathrm{~B}(\neg S)$ ), in opposition to the external version, which reads $\neg(\mathrm{B}(\mathrm{S}) \& \neg \mathrm{B}(\mathrm{S}))$. Although an internal contradiction is harmless in the case of quasitruth and most modal logics, external contradictions typically lead to trivialization. 
As an illustration of this problem in the practice of science, we mention an episode where we lack information; it concerns what was said by Julius Cesar just before he died. We currently have insufficient information to claim that he said "You too, child?", to Brutus ("Kai $\sigma \dot{v}, \tau \dot{\varepsilon} \kappa v 0 \nu$ ", in Greek), and we also lack definitive information to hold that he has said nothing at all. The reports (mainly of Plutarch and Suetonius) about Julius Cesar's assassination in the Senate in March 15th of 44 B.C. are not conclusive. As we are pointing in this section, in the absence of sufficient information, the quasi-truth theory implies that historians would have to believe (or have some kind of "pragmatic knowledge") of both possibilities registered. However, historians generally prefer to hold just one of the rival interpretations, while relinquishing the other, and provide partial reasons to ground their beliefs. For instance, those believing that Julius Cesar said nothing in the moment of his death justify their position by claiming that, although Suetonius reported that people by the time of the event stated that Cesar's last words in Greek were "kai $\sigma \dot{v}, \tau \dot{\varepsilon} k v 0 v$ ", Suetonius himself doubts that popular belief; besides, one could also justify this belief by using Plutarch's report, according to which Cesar did not say anything before dying, but merely pulled his toga over his head when he saw Brutus among the conspirators. In this case, someone defending this view would hardly claim to belief or to hold as somehow true the contradictory thesis, even though not having enough evidence to completely justify her position and raise it to the point of being a consensus in the community.

\subsection{Limitations OF THE FORMALISM}

In this topic we deal with what may be perceived as "limitations of the formalism". We will not necessarily present "problems" with the quasi-truth formalism, but we will highlight features of historical sciences that are not captured by the quasi-truth theory. In general, it is not the purpose of quasi-truth approach to deal with such features, but we pull them because they also bring to the fore some important properties that formal approaches should attempt to account for. That is, these properties are interesting for future extensions to the theory of quasi-truth or some concurrent formal theory that captures dynamics to scientific knowledge.

i) The formalism is not suitable to allow that new information (or new knowledge) leads us to revise what is already taken as known.

When we accept a set $\mathrm{P}$ of sentences as true from the start, we make it impossible that a revision of the currently accepted data could be made in the light of new evidence. This happens because new evidence cannot change the status of the propositions in P and also, it cannot change the status of properties and relations already known, i.e., the elements in the first and second members in the extension of predicates and relations cannot be changed in an extension. In fact, it is enough to remember that the formalism demands that that which counts as new information and evidence should always be consistent with $\mathrm{P}$, and also, that the formalism demands the total structures extend, but do not modify, the extensions of the first and second members of the interpretations of properties and relation symbols (those that are defined as known; again, see our comments about that feature in section 2). In these terms, there is a fixed nucleus of a partial structure that cannot be modified.

This rigidity does not reflect the openness and incompleteness of scientific knowledge (neither in historical sciences, nor in physical sciences). In particular, data are, sometimes, 
much more open to revision in human sciences, and much of what we consider now a datum could be revised in the light of new evidence; new discoveries could change even what is sometimes taken as a consensus of the community of researchers. This kind of progress, which we mentioned in the previous section, allows us to eliminate certain accounts of the past as implausible in the light of new evidence. However, it is precisely this kind of movement that is forbidden by the quasi-truth approach. Once an account is chosen, one can only add to it.

There are several examples of twists in historiography. We will mention two here, listed from the weakest to the strongest. The first example (I) is a case where the appreciation of new information has led to a revision of factual interpretation in art history; the second example (II) is a case in which from an empirical study of a historical phenomenon it created a methodological revolution and several historical phenomena had to be revised:

(I) Greco-Roman sculptures have long been believed to be intentionally monochromatic. Culturally, this not only affected our knowledge of Greece, but also our actions in relation to this knowledge: it fatally influenced the sculptures of various artistic movements in the West, especially in the Renaissance and the neoclassicism. In addition, historiographical interpretations were built with this premise. In 1764 Johann Winckelmann-often considered the "father of art history"wrote that "white is the color that shows the most rays of light, and thus is most easily perceived." Because of this, he believed that "a beautiful body will be all the more beautiful the whiter it is." Evidently such a point of view of Winckelmann also reflected a case of racism, but still he took this point seriously and his aesthetic position was very influential among historians, and even among some philosophers, like Hegel. We now know, however, that the Greek sculptures were highly polychrome (with colors that were lost over time). The beauty in the saturation of the sculptures is attested in Greece in some ancient texts, as in a passage by Helena from Euripides- "If only I could shed my beauty and assume an uglier aspect $[. .$.$] the way you would wipe color off a statue." - and among the Romans$ the coloring process (whose pigments were extracted from minerals, beeswax and egg yolks) is detailed by Vitruvius and Pliny the Elder. Moreover, perhaps the most fatal information for Winckelmann's interpretation is that it is now possible to know the colors of yore in Greek sculptures by exposing them to ultraviolet light (Brinkmann, 2008, pp. 18-39).

(II) Here is perhaps the most famous example in historiography: the case of the Donation of Constantine (Constitutum Donatio Constantini or Constitutum domini Constantini imperatoris), which is a document presented in the Middle Ages as a Roman imperial edict (Vauchez, 2001, p. 445). According to the content of the donation, the emperor confessed his faith, told that he had been cured of leprosy by the Pope's intercession before his conversion and gave the Pope authority over the Christian communities of the East (Antioch, Jerusalem, Alexandria, and Constantinople). Lateran, St. Peter, and St. Paul's churches-outside the walls of Rome-lands at various points in the Roman Empire in Judea, Greece, Thrace, and Asia Minor, and gave the Pope the faculty of raising the senators of the empire to the level of priests. In the fifteenth century, however, Lorenzo Valla presented a series of philological documents and arguments that would prove the 
in-authenticity of the donation document. Valla's work over time has not only meant an attempt to destabilize the authority of the clergy, but-for historians since then-has highlighted the importance of questioning documents and gathering information that involves language transformations over time and the need to establish an identity relationship between the document and the time when it would have been produced.

We may indicate what is going on in the examples discussed above as follows: even if the formalism of quasi-truth is attempting to capture a kind of dynamics when distinct extensions of our actual knowledge are allowed and taken into account, this dynamics does not allow that we revise what is already known or taken as known. The dynamics involved in the formalism allows for the accumulation of information, but does not allow for the revision of previously accepted information (see ex. I); that holds also for theories (see ex. II, if understood as a set of information describing broader phenomena) and for changes in methodology which accounts for the obtaining of new information (and even of information of a different nature), which were previously ignored.

This limitation is somehow intrinsic to the quasi-truth project, which seems to formalize knowledge and its progress by additions, without revision or change, be them partial or radical. As it is put by da Costa and French (2003, pp. 13-14):

If the final conception is taken to be complete or total, then our conception at any given time prior to the realization of this limit may be said to be partial. And because it is, at any given time, partial, it is, at that time, open in the sense that it may be completable in a variety of ways.

The idea underlying the approach suggested seems to be that there is a final conception, which is reached through progressive stages. These progressive stages are, on their turn, stages of addition of information to the partial information or partial knowledge we have, on their way to the total knowledge. The dynamics of knowledge proceeds by addition, without space for revision or correction. In contrast, dynamic epistemic logics, such as Public Announcement Logic (van Ditmarsch; Kooi, 2008, cap. 4), are examples of logical systems that traditionally deal with revising beliefs and knowledge, and such reviews can be done step by step, also responding to inadequacy (see 4.1.ii). However, in its usual formulation, it does not have some important properties of the Quasi-Truth Theory.

One could attempt to open space for accommodating this kind of situation by allowing that even the members of the first or the second component of a partial relation could be changed when extensions of a given sps are made. That, however, would change significantly the definition of quasi-truth, given that one can no longer consider that normal structures are extensions of the original sps (indeed, they change the sps in significant ways, allowing that the extension of any predicate change in any component). It is hard, to say the least, what would remain of quasi-truth in this picture, so that perhaps this is not the most appropriate move.

Another option would be to follow the suggestion advanced by Tohmé et al. (2011). They suggest, in a nutshell, that one may provide a kind of dynamics for partial structures, accommodating revision of knowledge, by fixing the locus of change in the set of accepted sentences $\mathrm{P}$ in a given sps A (and they suggest that this would combine nicely with defeasible reasoning, a claim we have no space to discuss here). The plan is that once new information is obtained, even information requires some revision of previous knowledge, it 
may be incorporated in $\mathrm{P}$, and this would amount to a change in the set of available normal structures that extend A (see in particular Tohmé et al., 2011, p. 57). Something similar holds for the case where we are recommended to eliminate some of the sentences in P; it also leads to a change in the number of available extensions of the sps. That is, inserting or eliminating constraints in $\mathrm{P}$ leads to the set of available extensions becoming dynamic, and as a result, to the set of sentences that are quasi-true and quasi-false. This would even allow us to revise some of the knowledge already available. To illustrate the proposal, consider the case of the whiteness of the Greco-Roman sculptures we have just discussed. The plan is that once we discover that they were indeed colored, and not white, we may insert that information in P. This insertion blocks extensions of the structures where sculptures are treated as white, or where whiteness plays a role. This brings a dynamics to partial structures, and allows for a kind of defeasibility in the use of pragmatic truth.

Now, although this is a promising direction in which to extend the pragmatic truth approach, we fear it puts too much weight in the set $\mathrm{P}$, and downplays the importance of the actual partial relations that are constituting the structure, which are, in the original proposal, responsible for the representation of the dynamics of lack of information. That is, $\mathrm{P}$ is to be an auxiliary set, and the important part is played by the partial relations. If we let $\mathrm{P}$ do the heavy work on the dynamics, then, with no constraints, it may be used in ad hoc ways to accommodate any kind of change. Also, this approach does not address our concern directly. Our discussion concerns mostly revision of the elements of the first and second members of the partial relations (those elements of the extension of partial relations that are fixed once and for all in a pragmatic structure). That is, the kind of dynamics we find necessary would have to account for changes in partial relations that are settled as known to hold or known not to hold, something the proposal we are discussing does not address. The proposal under examination considers only how changes in $\mathrm{P}$ affect the dynamics of the third members of a partial relation; it suggests that once $\mathrm{P}$ is changed, we also change the way the third members of a partial relation may be moved in the resulting extensions of the pragmatic structure (recall, because changing P changes the set of available extensions). So, the proposal does not really address our concern here, although it provides for important advances, of course.

ii) Counterfactual histories, or alternative histories, cannot be appropriately taken into account by the formalism of quasi-truth. One cannot change the collection $\mathrm{P}$ of the sentences that are accepted beforehand, given that these sentences inform and somehow put a limit to the allowable extensions of the sps in which we evaluate our sentences. This puts a barrier on the kinds of historical investigations that can be accommodated by the apparatus of quasi-truth.

Throughout the twentieth century, the notion of counterfactuality gained an important place in the relationship between historical science and literature (Maar, 2014). In historiography, we highlight the emergence of the area of Virtual History (Hawthorn, 1991) —an area dedicated to studying causal relations between historical events through counterfactual conjectures-and the Speculative Evolution that seeks to build models of what certain species would be like in the past if certain things had not occurred (and other things had happened in their place) or even how certain species that will exist today over the next thousands of years will evolve. In addition, counterfactuality has been used by historians of historiography to characterize causality in the historical sciences. In this characteriza- 
tion, if there were no other (counterfactual) past, all past events would necessarily have occurred. However, this statement not only contradicts our intuition about some historical events-especially those that depended on human deliberation - but also trivializes the efforts of historians in the elaboration of explanatory statements (statements that begin with 'why', 'therefore' etc.): are historians simply always proving that what happened could only be that way? If we say no, that is, things could have been different (and indeed many historians seem to agree with this in various historical events), and we accept some version of the theory of counterfactual causal explanation, then the approach of quasi-truth will also leave out several important statements about which in historiography it is said to have knowledge or at least a consensus. Certainly, this is not something that the formalism of quasitruth aimed at representing, but, as mentioned, it is an important feature of current historiography. In this sense, it is something that formal approaches could also attempt to model and take into account.

Let us look at a classic example in the historiography given by Polybius of Megalopolis (2nd century BC) who already distinguished what he considered the "beginning" (arkhàs) of a historical process from the "cause" (aitia) of this process (Polybius, III, 6). Polybius does not present a counterfactual reading of causation; in his text, causality seems to be associated with the intentions of historical agents, but its distinction also serves our purposes. Polybius tells us that the war between Macedonia and the Persians would have started after the crossing of Alexander the Great into Asia, but his father, Philip of Macedonia, in the form of a planned action had actually caused it to expand his empire. Suppose a sentence $S$ designating "Philip's plans caused war against the Persians" and such a sentence is taken as historical knowledge. Thus, by the approach of the quasi-truth, this sentence is quasi-true (or "pragmatically true"), as we have seen, in relation to a single (real) past where it is possible that indeed Philip's plans caused the war. It turns out that the term 'caused' in the above sentence goes beyond the scope of the actual past. It is necessary to consider other possible pasts (in a modal logic) to verify or falsify $S$; otherwise we would lose the distinction between beginning and cause (in a counterfactual sense). Now, the influence of this distinction on the historical sciences certainly underlies many other sentences which are regarded as historical knowledge, and all of these seem to serve as counterexamples of historical knowledge that are not properly designated as quasi-true. In fact, given the importance of historical knowledge of a causal type, although the proponents of the quasi-truth approach do not consider a treatment by counterfactual models in their original approach, and although explanatory sentences and descriptive sentences enter without distinction in the quasi-truth formalism, they would nevertheless have to formulate some extension to account for these sentences, but without reducing them, for example, to merely descriptive sentences that answered "what or when happened" and not "why it happened", since, apparently, when we say why something happened, we are not talking about $a$ fact, but about something of a higher level, something about a fact that differentiates, for example, facts that occurred "by chance" or "by accident" and others that occurred because it had to be so or because there was a great probability that this was the course of history.

iii) As it is currently conceived, the language of quasi-truth theory is restricted to the interpretation of sentences attributing simple quasi-truth to a sentence, without any iteration of quasi-truth and mixing with temporal and epistemic operators. A simple example indicates that such mixing of quasi-truth with such operators is 
of utmost importance, as in "it's quasi-true 'that it was quasi-true that p", where p can be a proposition; for example, "the earth revolves around the sun". Propositions like that are very common in History of Science and other areas of the Intellectual History. A context of application of the above sentence appears when we say that Aristarchus anticipated Copernicus's theory when, in his time, the dominant idea was that the earth revolved around the sun. In general, these sentences are interpreted in epistemic and temporal logics as KPKp ("we know we knew that p"; PK = 'we knew' or 'it was the case that we know'). However, if the theory of quasi-truth formally represents the meaning of pragmatic truth, even if partially, then it should be able to interpret sentences as these.

It should be noted that this limitation of the formalism is not to be confused with the inadequacy of the formalism already hinted at, concerning the lack of possibility of revision of what is already taken as known. In fact, the formal inadequacy that we are now considering makes it difficult to interpret what is meant by "we knew that p (but no more)". In order to check that there are two distinct problems, consider that easily a language L can satisfy the previous formal inadequacy without dealing with the present formal limitation, and vice versa. It's possible to devise a language $\mathrm{L}_{1}$ with a dynamic logic system where the truth values of propositions such as $\mathrm{Kp}$ can be changed without, however, the language $\mathrm{L}_{1}$ including time modal operators such as P for "was the case that" (allowing, thus, for revisions of known propositions without allowing for expressions important for the History of Science). On the other hand, it is possible for an $\mathrm{L}_{2}$ language to allow formulas such as KPKp without, however, revising truth values of known propositions (dealing thus with problems in the History of Science, without dealing with revision of established knowledge). The language of quasi-truth systems, in turn, contains both problems.

\section{Conclusion}

In this paper, we have attempted to test the quasi-truth conceptual apparatus in its ability to accommodate incomplete knowledge. In particular, we have used cases and examples from the historical sciences to make our case more vivid, and illustrate the kind of problems we still find with the quasi-truth approach. Just as it happens to other sciences, such as physics, chemistry and biology, historical sciences present diverse cases where it may be claimed that there is openness and incomplete knowledge. In search for formal representation of historical knowledge, then, one cannot just stop at quasi-truth and hope to have the work done; rather, a formalism that accommodates the problems we have raised must be sought.

As we have argued, the apparatus of quasi-truth seems ill-suited to deal with some of the demands of incomplete information, both in general, as well as in the historical sciences. These failures come from the formulation of the formal apparatus of quasi-truth. Basically, the difficulties emerge from the fact that open information is represented by those sentences that may be quasi-true and also have a quasi-true negation; in modal terms, as those sentences that are possible, with a negation that is also possible. This engenders difficulties with the modal status of the sentences that are not thought of as 'open' (they cannot be revised, they end up being necessary), and of those that are open (they cannot be necessary). Also, a further pernicious consequence concerns the fact that the openness dealt with 
by the quasi-truth apparatus allows only for completing, not for revising, sentences that are already thought to be known. In this sense, it seems impossible to revise our knowledge available in the light of new information.

The apparatus of quasi-truth is also blind to a peculiar branch of the historical sciences: virtual/ counterfactual history. The problem, again, is that one cannot change the sentences that are taken as known (basically, those in P). But in order to make counterfactual history, one needs to do precisely that. This closes one important route of historical investigation. A formalism able to deal with such specifics is again desirable.

Given these limitations, it seems, we are better advised to look for distinct formal apparatuses if we are willing to deal with the incompleteness in historical sciences. Perhaps distinct apparatuses will be worth for distinct aspects of the incomplete knowledge in history. But that is still open, and we leave this discussion for a further work.

\section{Acknowledgments}

We would like to express our gratitude to two anonymous referees for their critical remarks on a previous version of this paper. Their comments contributed to the improvement of the arguments presented here.

\section{REFERENCES}

Arenhart, J. R. B., Krause, D. (2019). Quasi-truth and defective knowledge in science: A critical examination. Retrieved from <http://philsci-archive.pitt.edu/16356/>.

Brinkmann, V. (2008). The polychromy of ancient Greek sculpture. In R. Panzanelli, E. Schmidt \& K. Lapatin (eds.) The color of life: Polychromy in sculpture from antiquity to the present. Los Angeles, California: The J. Paul Getty Museum and the Getty Research Institute.

Bueno. O. (1999). Truth, quasi-truth and paraconsistency. Contemporary Mathematics 39, 275-293.

Bueno, O., \& da Costa, N. C. A. (2007). Quasi-truth, paraconsistency, and the foundations of science. Synthese 154, 383-399.

Bueno, O., \& De Souza, E. (1996). The concept of quasi-truth. Logique et analyse 39(153-154), 183-199.

Burgess, J. P. (1984) Basic tense logic. In D. Gabbay \& F. Guenthner (eds.). Handbook of philosophical logic. Volume II: Extensions of classical logic (pp.89-133). London: Kluwer Academic Publishers.

Carnielli, W., \& Rodrigues, A. (2019). An epistemic approach to paraconsistency: a logic of evidence and truth. Synthese 196(9), 3789-3813.

Coniglio, M., and Silvestrini, L. H. (2014). An alternative approach for quasi-truth. Logic Journal of the IGPL 22(2), 387-410.

Contessa, G. (2006). Scientific models, partial structures, and the new received view of theories. Stud. Hist. Phil. Sci. 37, 370-377.

Currie, A. (2019). Scientific knowledge and the deep past: History matters. Cambridge: Cambridge University Press.

da Costa, N. C. A. (1999). O conhecimento cientifico. São Paulo: Discurso Editorial.

da Costa, N. C. A., \& French, S. (2003). Science and partial truth: A unitary approach to models and scientific reasoning. Oxford: Oxford University Press.

da Costa, N. C. A., Krause, D., \& Bueno, O. (2006). Paraconsistent logic and paraconsistency. In D. Jacquette (ed.) Handbook of the philosophy of science. Volume 5: Philosophy of logic (pp. 655-775). Amsterdam: Elsevier. 
Danto, A. C. (1962). Narrative sentences. History and Theory 2(2), 146-179.

Danto, A. C. (2007)[1985] Narration and knowledge. (Including the integral text of Analytical Philosophy of History.) With a new introduction by Lydia Goehr and a new conclusion by Frank Ankersmit. New York: Columbia University Press.

Doležel, L. (1998). Worlds of fiction and history. New Literary History 29(4), 785-809.

Gallie, W. B. (1984). Explicações em história e as ciências genéticas. In P. Gardiner (ed.), Teorias da história (pp. 472-493). Lisboa: Fundação Calouste Gulbenkian.

Hart, K. H. (2018). Why do people still think that classical sculptures were meant to be white? Artsy 10, 2018 (5:25pm). Retrieved from <https://www.artsy.net/article/artsy-editorial-people-classical-sculptures-meant-white>.

Hawthorn, G. (1991). Plausible worlds: possibility and understanding in history and the social sciences. Cambridge: Cambridge University Press.

Krause, D., \& Arenhart, J. R. B. (2017). The logical foundations of scientific theories: Languages, structures, and models. New York: Routledge.

Little, D. (2010). New contributions to the philosophy of history. New York: Springer.

Lo Guercio, N., \& Szmuc, D. (2018). Remarks on the epistemic interpretation of paraconsistent logic. Principia 22(1), 153-170.

Lopes, J. L. (1992). «Tempo = Espaço = Matéria». In A. Novaes (ed.), Tempo e história (pp. 167-175). São Paulo: Companhia das Letras.

Maar, A. (2014). Possible uses of counterfactual thought experiments in history. Principia 18(1), 87-113.

Martínez-Ordaz, M. (2020). The ignorance behind inconsistency toleration. Synthese. Advance online publication. doi https://doi.org/10.1007/s11229-020-02593-4

Polybius (1962) [1889]. Histories (Evelyn S. Shuckburgh, trans). London, New York: Macmillan. Reprint Bloomington.

Tohmé, F., Delrieux, C., \& Bueno, O. (2011). Defeasible reasoning + partial models: a formal framework for the methodology of research programs. Foundations of Science 16, 17-45.

Van Ditmarsch, H., van der Hoek, W., Kooi, B. (2008). Dynamic epistemic logic. Dordrecht: Springer.

Vauchez, A. (2001). Encyclopedia of the middle ages. London: Routledge.

Wilson, T. V. (2008). How do scientists know if dinosaur fossils are male or female? In howstuffworks, Science, Nov. 6, 2008. Retrieved from <https://science.howstuffworks.com/environmental/earth/ geology/dinosaur-fossil-male-female1.htm >.

Jonas R. Becker Arenhart is associate professor at the philosophy department of the Federal University of Santa Catarina (Brazil). His main areas of research include the philosophy of logic and the metaphysics of science, in particular, the metaphysics of quantum mechanics. Recent publications include the book The Logical Foundations of Scientific Theories: Languages, Structures, and Models, in co-authorship with Décio Krause, published by Routledge.

AddRess 1): Department of Philosophy. Federal University of Santa Catarina. Campus Universitário Reitor João David Ferreira Lima. Trindade - Florianópolis. Santa Catarina - Brazil, 88040-900. e-mail: jonas. becker2@gmail.com

ORCID: 0000-0001-8570-7336

AddREss 2): Research Fellow at the Institute Vienna Circle (IVC). University of Vienna. Alserstrasse 23 Top 32 (1080 Vienna, Austria)

Vítor Medeiros Costa is a doctoral student at the philosophy department of the Federal University of Santa Catarina (Brazil). His main areas of research include modal logics and philosophy of science, in particular, the philosophy of historical sciences. Currently works with applications of temporal logic and dynamic logic to understand historical knowledge.

Address: Department of Philosophy. Federal University of Santa Catarina. Campus Universitário Reitor João David Ferreira Lima. Trindade - Florianópolis (Santa Catarina - Brazil, 88040-900). e-mail: vitor.m.costa@outlook.com

ORCID: 0000-0002-8719-4025 